Astrophysical Journal, in press

\title{
Comparisons of the Interstellar Magnetic Field Directions obtained from the IBEX Ribbon and Interstellar Polarizations
}

\author{
Priscilla C. Frisch \\ Dept. Astronomy and Astrophysics, University of Chicago, Chicago, IL 60637 \\ frisch@oddjob.uchicago.edu \\ and \\ B-G Andersson \\ SOFIA, USRA \\ bganderssonesofia.usra.edu \\ and \\ Andrei Berdyugin \\ Tuorla Observatory, University of Turku, Finland \\ andbereutu.fi \\ and \\ Herbert O. Funsten \\ Los Alamos National Laboratory, Los Alamos, NM \\ hfunstenelanl.gov \\ and \\ Mario Magalhaes \\ Inst. de Astronomia, Geofisica, University de Sao Paulo, Brazil \\ mariodastro.iag.usp.br \\ and
}


David J. McComas ${ }^{1}$

Southwest Research Institute, San Antonio, TX

DMcComaseswri.edu

and

Vilppu Piirola

Tuorla Observatory, University of Turku, Finland

$$
\begin{aligned}
& \text { piiroladutu.fi } \\
& \text { and }
\end{aligned}
$$

Nathan A. Schwadron

Space Science Center, University of New Hampshire

nschwadron@guero.sr.unh.edu

and

Jonathan D. Slavin

Harvard-Smithsonian Center for Astrophysics, Harvard, Cambridge, MA

$$
\begin{gathered}
\text { jslavinecfa.harvard.edu } \\
\text { and }
\end{gathered}
$$

Sloane J. Wiktorowicz

Astronomy Dept., University of California, Berkeley

$$
\text { sloane@berkeley .edu }
$$

\section{${ }^{1}$ also University of Texas, San Antonio, TX}

ABSTRACT 
Variations in the spatial configuration of the interstellar magnetic field (ISMF) near the Sun can be constrained by comparing the ISMF direction at the heliosphere found from the Interstellar Boundary Explorer spacecraft (IBEX) observations of a 'Ribbon' of energetic neutral atoms (ENAs), with the ISMF direction derived from optical polarization data for stars within $\sim 40 \mathrm{pc}$. Using interstellar polarization observations towards $\sim 30$ nearby stars within $\sim 90^{\circ}$ of the heliosphere nose, we find that the best fits to the polarization position angles are obtained for a magnetic pole directed towards ecliptic coordinates of $\lambda, \beta \sim 263^{\circ}, 37^{\circ}$ (or galactic coordinates of $\ell, b \sim 38^{\circ}, 23^{\circ}$ ), with uncertainties of $\pm 35^{\circ}$ based on the broad minimum of the best fits and the range of data quality. This magnetic pole is $33^{\circ}$ from the magnetic pole that is defined by the center of the arc of the ENA Ribbon. The IBEX ENA ribbon is seen in sightlines that are perpendicular to the ISMF as it drapes over the heliosphere. The similarity of the polarization and Ribbon directions for the local ISMF suggest that the local field is coherent over scale sizes of tens of parsecs. The ISMF vector direction is nearly perpendicular to the flow of local ISM through the local standard of rest, supporting a possible local ISM origin related to an evolved expanding magnetized shell. The local ISMF direction is found to have a curious geometry with respect to the cosmic microwave background dipole moment.

Subject headings: ISM: magnetic fields, clouds, HI — solar system: general — stars: winds, outflows

\section{Introduction}

The recent unexpected discovery by the Interstellar Boundary Explorer mission (IBEX, McComas et al. $2009 \mathrm{~b}$ ) of a 'Ribbon' of energetic neutral atoms (ENA) provides the first direct evidence, and indirect measurement, of the interstellar magnetic field embedded in the low density partially ionized cloud surrounding the Sun (McComas et al. 2009a; Fuselier et al. 2009; Funsten et al. 2009; Schwadron et al. 2009; Möbius et al. 2009). The IBEX Ribbon is visible at 1 AU for directions where the sightline is perpendicular to the interstellar magnetic field draping over the heliosphere. The center of the Ribbon arc defines a value for the direction of the interstellar magnetic field (ISMF) in the interstellar material (ISM) that is interacting with the heliosphere. The ISMF directions traced by the IBEX Ribbon, and weak interstellar polarizations observations of towards nearby stars, together provide a unique constraint on the local ISMF direction, the outer boundary conditions of the heliosphere, and the global magnetic turbulence in the low density interstellar material near the Sun. In this paper we compare the available data on the ISMF direction from optical polarization of local starlight, with the ISMF direction signified by the IBEX Ribbon. 
Interstellar material (ISM) sets the outer boundary conditions of the heliosphere, including the thermal and ram pressures of the gas, and magnetic pressures (Davis 1955; Parker 1961; Holzer 1989). The heliosphere nose is defined by the upwind direction of the flow vector of interstellar gas through the heliosphere, and is $15^{\circ}$ above the galactic center and $5^{\circ}$ above the ecliptic plane. The $0.05-6 \mathrm{keV}\left(\sim 50-1000 \mathrm{~km} \mathrm{~s}^{-1}\right)$ ENAs detected by IBEX are formed in regions where charge-exchange between interstellar neutral hydrogen and energetic ions create inward flowing particles that reach the IBEX detectors. The ENAs formed inside the solar wind termination shock propagate away from the Sun, but the subsonic solar wind and pickup ions outside of the termination shock, with momentum components directed back towards the Sun, can create the ENAs measured in the inner heliosphere by IBEX (e.g. Izmodenov et al. 2009; Heerikhuisen et al. 2008). The subset of ENAs that propagate back towards IBEX at 1 AU are proving an excellent diagnostic of the heliosphere boundary conditions. The center of the Ribbon arc indicates an interstellar magnetic field (ISMF) direction towards ecliptic coordinates $\lambda, \beta=221^{\circ}, 39^{\circ}$ (or in galactic coordinates towards $\ell, b=33^{\circ}, 55^{\circ}$, Funsten et al. 2009; Fuselier et al. 2009). The Ribbon's arc does not follow the equator of the interstellar magnetic field (ISMF) defined by the arc center, but rather traces the distortion of the ISMF by the heliosphere (Schwadron et al. 2009). For magnetic field $B$ and radial direction $R$, the length and width of the $B \cdot R$ region depend on magnetic pressure and the limit set on $B \cdot R$, respectively. The Ribbon latitude, in comparison to the magnetic pole defined by the arc center, depends on the distance beyond the heliopause of the Ribbon formation.

The ISM in the low column density sky is formed into filamentary anisotropic structures, whether it is neutral gas seen in the $21-\mathrm{cm} \mathrm{H}^{\mathrm{o}}$ line, ionized gas observed in $\mathrm{H} \alpha$ emission, or dust traced by infrared emission or the optical polarizations of starlight. Magnetic fields are a candidate force for controlling this observed filamentary structure, since they generate anisotropic forces on the ISM through pressure that acts perpendicular to the field lines, and tension that acts parallel to the field lines (Heiles 2009). Prior to the heliospheric diagnostics of the very local ISMF, such as the IBEX Ribbon, the only data capable of constraining the ISMF within $\sim 50$ pc were highsensitivity measurements of the polarization of starlight by magnetically aligned interstellar dust grains. The IBEX Ribbon is a feature that can be simulated with full heliosphere models, that simultaneously give the pressure of the ISM, and the orientation and strength of the ISMF. In the upwind direction (near the galactic center), the Sun appears to be at or close to the boundary of the surrounding cloud (Frisch et al. 2009). The ENA production simulation of Heerikhuisen et al. (2010), which is unconfirmed, predicts that IBEX is capable of detecting variations in the Ribbon configuration that would be caused by variations in the interstellar density or magnetic field, such as expected when the Sun exits the cloud now around the heliosphere (Frisch et al. 2010). The relation between the ISMF directions traced by the IBEX Ribbon, and the observations of the weak interstellar polarizations of light from nearby stars, provides a powerful tool for understanding both the distortion of the ISMF at the heliosphere, and deviations from a homogeneous ISMF field over 
the nearest tens of parsecs.

The magnetic configuration traced by the ENA ribbon, together with the asymmetry of the heliosphere, is a function of angle between the interstellar gas flow vector and the ISMF direction. The flow of interstellar $\mathrm{He}^{\circ}$ through the heliosphere was measured by the Ulysses probe, and showed a warm cloud $T \sim 6300 \pm 340 \mathrm{~K}$, with velocity $26.3 \mathrm{~km} \mathrm{~s}^{-1}$ and an upwind direction towards $\lambda, \beta \sim 255.5^{\circ}, 5.1^{\circ}$ (or $\ell, b=3.6^{\circ}, 15.1^{\circ}$, Witte 2004, after converting to $\mathrm{J} 2000$ coordinates). The Ribbon data therefore indicate an angle of $\sim 46^{\circ}$ between the $\mathrm{He}^{\circ}$ flow velocity and field direction. The gaseous component of the heliosphere boundary conditions has been determined from models of the cloud ionization state based on interstellar absorption lines, which indicate that the surrounding interstellar gas is partially ionized with hydrogen $\sim 25 \%$ ionized, and low density, $n_{\text {tot }} \sim 0.27 \mathrm{~cm}^{-3}$ (Slavin \& Frisch 2008, e.g. model 26). The ratio of thermal and magnetic pressures, $\beta$, is unknown for the surrounding cloud. If the local ISM is near the boundaries of an evolved superbubble, which is not yet clear, $\beta \sim 1$ might be expected. For the assumption of $\beta=1$, the interstellar field strength is $\sim 2.8 \mu \mathrm{G}$ (model 26). For comparison, Zeeman splitting of $\mathrm{H}^{\mathrm{o}} 21-\mathrm{cm}$ lines show that superbubble shells with filamentary structures have high ISMF strengths and small $\beta<0.1$, although column densities are over 100 times values in the local ISM (Heiles 1989). Low $\beta$ values are also found for the Local Bubble walls foreground of the Coalsack Nebula based on a Chandrasekhar-Fermi analysis of the polarization component in the plane of the sky (Andersson \& Potter 2006).

The ISMF direction at the heliosphere is predicted by self-consistent heliosphere models (e.g. Ratkiewicz et al. 2000; Pogorelov et al. 2004, 2009; Opher et al. 2009; Izmodenov 2009) that are constrained by the interstellar neutral and plasma density in the circumheliospheric ISM (Slavin \& Frisch 2008), the 10 AU difference between the Voyager 1 and Voyager 2 measurements of the termination shock distance (of which 3-4 AU could arise from variable solar wind, Stone et al. 2008), and the $\sim 5^{\circ}$ offset between the directions of interstellar $\mathrm{H}^{\circ}$ and $\mathrm{He}^{\circ}$ flowing into the heliosphere (Witte 2004; Lallement et al. 2005, after precessing the $\mathrm{He}^{\circ}$ and $\mathrm{H}^{\mathrm{o}}$ directions into a common coordinate system). Heerikhuisen et al. (2010) models reproduce the IBEX Ribbon for an ISMF direction directed towards $\lambda, \beta \sim 223^{\circ}, 40^{\circ}$ (or $\ell, b \sim 35^{\circ}, 54^{\circ}$, hereafter $\ell, b$ are galactic coordinates). This direction is in good agreement with the ISMF direction $\ell, b \sim 30^{\circ} \pm 5^{\circ}, 33^{\circ} \pm 4.3^{\circ}$, for $B \sim 3.8 \mu \mathrm{G}$, from MHD models of heliospheric asymmetries of Ratkiewicz \& Grygorczuk (2008). Opher et al. (2009) use the heliosphere asymmetries from Voyager data and the $\mathrm{H}^{\mathrm{o}}-\mathrm{He}^{\mathrm{o}}$ offset to conclude that the ISMF field direction is inclined by $60^{\circ}-90^{\circ}$ with respect to the galactic plane. Several heliosphere models suggest the very local ISMF is directed towards the southern solar magnetic pole, such as Swisdak et al. (2010) who base their conclusion on the assumption that the $3 \mathrm{kHz}$ emissions detected by the Voyager satellites during the early 1990's are formed by reconnection. The general agreement between the ISMF determined from the ENA ribbon, the Heerikhuisen et al. model, and the Ratkiewicz \& Grygorczuk (2008) model suggest directions for 
the ISMF at the heliosphere near the center of the Ribbon arc.

The new Ribbon diagnostic of the ISMF at the heliosphere provides an opportunity to evaluate the large-scale distortion or turbulence of the local ISMF, e.g. over $\sim 40 \mathrm{pc}$, through comparisons with the polarization of starlight caused by magnetically aligned interstellar dust grains. Optical polarization data show that once the curvature of spiral arms is taken into account, the global ISMF beyond $\sim 0.3 \mathrm{kpc}$, is oriented towards $\ell \sim 83^{\circ}$ (Heiles 1996). Faraday rotation data show that the polarity of the global ISMF is directed towards $\ell \sim 83^{\circ}$ (Taylor et al. 2009). The Loop I magnetic superbubble, centered about $100 \mathrm{pc}$ away, is a local large distortion of the global field with an angular diameter of $\sim 160^{\circ}$ (e.g. Heiles 1976, 1998; Wolleben 2007). It is prominent in dust, gas, synchrotron emission, optical polarization, and Faraday rotation data. Loop I is the best candidate for the phenomena that links the ISMF at the heliosphere with the ISMF causing nearby star polarizations (Frisch 2010). The overall geometry of Loop I, and the 'S1' subshell, indicate that $\mathrm{S} 1$ has expanded to the solar location if it is approximately spherical, and the ISMF direction appears consistent with nearby polarization data for a field direction near $\ell, b \sim 71^{\circ}, 48^{\circ}$, with uncertainties of $\sim \pm 30^{\circ}$.

The ISM within $\sim 20$ pc of the Sun has very low column densities, typically $<10^{18.5} \mathrm{~cm}^{-2}$, and therefore low extinctions. Pioneering efforts to measure polarized starlight caused by magnetically aligned interstellar dust grains found that nearby space is very empty of the ISM, except for a patch of nearby dust, within $40 \mathrm{pc}$, primarily in the fourth Galactic quadrant between $270^{\circ}$ and $360^{\circ}$, and in the southern hemisphere (Piirola 1977; Tinbergen 1982). This dust 'patch' has a very local component, as the $2.5 \sigma$ detection of polarization towards the star $36 \mathrm{Oph}, 5 \mathrm{pc}$ beyond the heliosphere nose, shows. (It is remarkable that many of these weak polarizations line up along the IBEX Ribbon, see Fig. 1) The cloud giving rise to the polarization towards $36 \mathrm{Oph}$ is not the circumheliospheric ISM, since the velocities of the two clouds differs by $\sim 2 \mathrm{~km} \mathrm{~s}^{-1}$ (Frisch et al. 2009), so that the polarizations in this patch potentially trace a different ISMF direction than the Ribbon arc. The next set of studies of the polarization of nearby starlight was conducted by Leroy (1993), from the northern hemisphere. Leroy made a catalog of observations of 1000 stars within $50 \mathrm{pc}$, with different sensitivity levels, and reconfirmed the emptiness of nearby space. However, he was unable to either confirm or disprove the existence of the polarization patch observed by Tinbergen. More recently, polarization observations with high measurement accuracy have been made with the PlanetPol instrument, primarily in the northern sky (Bailey et al. 2010). The Tinbergen, Piirola, and PlanetPol data are used in this study.

The goal of this study is to test the ISMF direction indicated by the center of the arc of the IBEX Ribbon with the ISMF direction traced by the polarization of light from nearby stars, using mainly polarization data from the literature with a range of sensitivity levels. These results are preliminary, in the sense that more and better high-sensitivity polarization data may affect the 
conclusions.

\section{Local Magnetic Field Direction from Interstellar Polarizations}

Magnetically aligned dust grains in the ISM create a birefringent medium with lower opacities parallel to the ISMF direction (e.g., Davis \& Berge 1968; Lazarian 2003). The position angles of weakly polarized light, $\lesssim 0.02 \%$, from nearby stars can then be used to trace the local ISMF direction. A distance limit of $40 \mathrm{pc}$ is selected for polarization measurements of the local ISM, since the "boundary" of the Local Bubble is at about $50 \mathrm{pc}$ for galactic longitudes $300^{\circ}-360^{\circ}$ and positive latitudes (e.g. Barstow et al. 1997). We assemble polarization data for nearby stars, omitting stars with known intrinsic polarization or circumstellar disks, and systematically evaluate the polarization position angles for a grid of $i$ possible ISMF directions, $\hat{k}_{B, i}$, in order to determine the ISMF direction (e.g. rotated coordinate system) that provides the best alignment between polarization position angles and the meridians of the ISMF. The polarization data used for these comparisons consist of three sets of archival starlight polarization data, and new unpublished observations for three stars. The star sample is restricted to objects within $\sim 40 \mathrm{pc}$ of the Sun. The combined data sets of Tinbergen (1982) 1 and Piirola (1977) provide data on $\sim 140$ stars, at $1 \sigma$ sensitivity levels of $\sim 7 \times 10^{-5}$ degree of polarization (or equivalently $0.007 \%$ or 70 parts per million), and in both northern and southern hemispheres. The strongest polarizations are seen towards the heliosphere nose region, with strengths of $\sim 0.02 \%$. Recent PlanetPol measurements at sensitivities of a few parts per million by Bailey et al. (2010) add additional data in the northern galactic and ecliptic hemispheres, where polarizations are typically very weak $(<0.004 \%)$. Unpublished observations of several stars are also available, including of $\beta$ Oph (HD 161096), with polarization $P=0.00506 \pm 0.00008 \%$, and equatorial position angle $\theta_{\mathrm{RA}}=166.2^{\circ} \pm 0.8^{\circ}$ (S. Wiktorowicz; data acquired with the POLISH instrument, Wiktorowicz \& Matthews 2008), where $\theta_{\mathrm{RA}}$ is the polarization position angle in equatorial coordinates. We add measurements of polarizations towards $\lambda$ Sgr (HD 169916) and $\tau$ Sgr (HD 177716), that were acquired by A. Berdyugin and V. Piirola in the R-band with the DIPOL polarimeter (Piirola et al. 2005) on the KVA-60 remotely operated telescope. For $\lambda$ Sgr, $P=0.036 \pm 0.007 \%$ and $\theta_{\mathrm{RA}}=109^{\circ} \pm 7^{\circ}$. For $\tau$ Sgr, $P=0.028 \pm 0.007 \%$ and $\theta_{\mathrm{RA}}=113^{\circ} \pm^{\circ} 7$. There are several stars with data from multiple sources (e.g. $\lambda$ Sgr, $\tau$ Sgr, and $\beta \mathrm{Oph}$ ). In these cases all of the data were included with equal weight in the analysis.

The optical polarization position angles are plotted in galactic and ecliptic coordinates on

\footnotetext{
${ }^{1}$ During 1973-1974 when the southern hemisphere Tinbergen data were acquired, the solar magnetic polarity was north pole positive ( $\mathrm{A}>0$, field lines emerging at the north pole). The solar polarity should have no effect on the optical polarization, as long as the grains are truly interstellar and outside of the heliopause.
} 
Aitoff projections in Figs. 1, 2. The plotted size of the polarization vector is not related to the polarization strength, which spans over an order of magnitude for these stars (see Fig. 5). The position angle of a vector is defined with respect to the north-south meridian passing through the position of the object, with position angle increasing in the direction of increasing longitude.

To derive the orientation of the local ISMF we used a minimization procedure in which we assumed that the local ISMF has a dipole configuration, so that the variations in the orientations of the observed polarizations are due to the location of the field poles in the sky. If this assumption is correct, there should be a coordinate transformation that transforms all of the observed polarization vector directions into vectors that are parallel to a meridian of the transformed coordinate system. Following Appenzeller (1968), the position angle is transformed to coordinate system $\hat{k}_{B, i}$ using the relation:

$$
\cot \left(\theta_{\mathrm{RA}}-\theta_{\mathrm{i}}\right)=\frac{\cos \left(b_{\mathrm{i}}\right) * \tan \left(b_{\mathrm{N}}\right)-\cos \left(\ell_{\mathrm{i}}-\ell_{\mathrm{N}}\right) * \sin \left(b_{\mathrm{i}}\right)}{\sin \left(\ell_{\mathrm{i}}-\ell_{\mathrm{N}}\right)}
$$

where $\theta_{\mathrm{RA}}$ is the position angle in the equatorial coordinate system, and $\theta_{\mathrm{i}}, l_{\mathrm{i}}$, and $b_{\mathrm{i}}$ are the polarization position angle and star coordinates in the $\mathrm{i}^{\text {th }}$ coordinate system corresponding to $\hat{k}_{B, i}$. The north pole of the equatorial coordinate system is located at $l_{\mathrm{N}}, b_{\mathrm{N}}$ in the $i^{\text {th }}$ coordinate system. Eq. 1 is used to transform between both equatorial and ecliptic or galactic coordinates, and between equatorial coordinates and the rotated ISMF frame $\hat{k}_{B, i}$.

The ISMF direction that provides the best fit to the polarization position angles is selected by testing the polarization vector directions against the $\hat{k}_{B, i}$ grid of possible ISMF directions. It is assumed that the "correct" ISMF direction will be parallel to the polarization vectors. The grid of possible ISMF directions are spaced by $1^{\circ}$ in galactic longitude, $\ell$, and latitude, $b$, over the intervals $0^{\circ}<\ell_{\mathrm{i}}<360^{\circ}$ and $\left|b_{\mathrm{i}}\right|<85^{\circ}$. This comparison between polarization position angles and the ISMF direction was restricted to the subset of stars that have polarizations larger than $3 \sigma$, where $1 \sigma$ is the measurement uncertainty, and that are located within $\sim 90^{\circ}$ of the heliosphere nose. Since the heliosphere nose is $\sim 15^{\circ}$ from the galactic center, the fitting region is essentially restricted to the first and fourth galactic quadrants where ISM and the magnetic sky are dominated by the magnetic shell of Loop I. Polarization position angles for each star $j$ were first rotated into the $\hat{k}_{B, i}$ coordinate system using eq. 1 , to obtain the new position angle $\theta_{\mathrm{j}, \mathrm{i}}$ relative to the new ISMF direction corresponding to the grid point $\hat{k}_{B, i}$. Polarization position angles are degenerate with respect to the north and south meridian directions, and the values of $\theta_{\mathrm{j}, \mathrm{i}}$ were corrected to be between $0^{\circ}$ and $180^{\circ}$ by adding or subtracting $180^{\circ}$.

The best fitting ISMF then becomes the direction where the rotated ISMF (the $\mathrm{i}^{\text {th }}$ grid point) yields a minimum of some function that describes a good match between the rotated polarization position angles and the rotated field direction. The function for determining the best fitting ISMF 
that is adopted here is the direction where the ISMF pole corresponds to the $\mathrm{i}^{\text {th }}$ grid point that yields the minimum value for the function $F_{\mathrm{i}}$, where $F_{\mathrm{i}}=\overline{\sin \left(\theta_{\mathrm{j}, \mathrm{i}}\right)}$ is the mean sin of $j$ polarization position angles $\theta_{\mathrm{j}, \mathrm{i}}$ in the rotated coordinates. All data points are weighted equally, rather than by measurement accuracy, since otherwise data sampling the Ribbon region would have lower weights since the older data (from Tinbergen, 1982, see Fig. 1) have lower measurement accuracies. The position angle is the angle between the polarization vector and a meridian, so that for a perfect fit the rotated position angles will be $0^{\circ}$ or $180^{\circ}$, and $F_{\mathrm{i}}=0$. Figs. 34 shows the color-coded map of $F_{\mathrm{i}}$. The best fitting ISMF direction, $\hat{k}_{B \text {,best }}$, that is determined where the minimum of $F_{\mathrm{i}}=0.46$, is directed towards the ecliptic coordinates $\lambda, \beta \sim 263^{\circ}, 37^{\circ}$, or galactic coordinates $\ell, b \sim 38^{\circ}, 23^{\circ}$ (Table 1). The broad minima shown in Figs. 34, together with the range of data quality, suggest that a more accurate uncertainty for the best fit is $\pm 35^{\circ}$. Figs. 34 also displays (only) the polarization position angles that were used in the ISMF fitting process.

Several tests of the fitting process were made. When the stellar data set was restricted to stars within $35 \mathrm{pc}$, the best fit ISMF direction changed by $\sim 10^{\circ}-20^{\circ}$ because four measurements near the ecliptic equator were removed from the sample, leaving a bimodal sample biased towards stars in the northern ecliptic hemisphere. The fitting function based on $F_{\mathrm{i}}^{\prime \prime}=\overline{\sin \left(\theta_{\mathrm{j}, \mathrm{i}}^{2}\right)}$ was also tried, and it gave a best-fitting ISMF direction towards $\ell, b=53^{\circ}, 25^{\circ}$, however this function overweights position angle values near the equator of the rotated ISMF, and so seems less suitable for this small set of data. The fitting procedure was repeated by varying the initial coordinate system used in the fit (e.g. equatorial, ecliptic, or galactic), and the results agreed to within $\sim 1^{\circ}$. Another check was made by omitting the step of converting the rotated $\theta_{\mathrm{j}, \mathrm{i}}$ values to the range of $0^{\circ}-180^{\circ}$, yielding as expected the same result.

The polarization position angles in the rotated frame have been constrained to be between $0^{\circ}$ and $180^{\circ}$, so that the best fit value $F_{\min }=0.46$ corresponds to mean position angles of $27^{\circ}$. When the standard deviation of the position angles is included, the best-fit mean position angle in the rotated frame is $27_{-19}^{+23}$ degrees. In principle the dispersion in the position angles for the best-fitting ISMF direction could either be due to variations in the global configuration of the nearby ISMF over scale-lengths comparable to typical scales of energy injection, or to small scale turbulence at scale lengths typical of the plasma and magnetic properties of the partially ionized gas. However the intrinsic measurement accuracies of the data from the northern versus southern hemisphere data sets differ substantially, so that understanding small scale magnetic turbulence will require higher precision data in the southern hemisphere.

For a uniform distribution of interstellar dust near the Sun, and constant grain alignment efficiency, the polarization strengths will increase as the angular distance between the star and the poles of the ISMF increase, i.e. the polarizations are strongest where the sightline is more perpendicular to the ISMF direction. These data do not show such an effect (Fig. 5). Instead, 
Table 1. Best-fitting Magnetic Field Pole ${ }^{1}$

\begin{tabular}{lcc}
\hline \hline $\begin{array}{l}\text { Coordinate } \\
\text { System }\end{array}$ & Longitude & Latitude \\
\hline \multicolumn{2}{l}{$\begin{array}{l}\text { Polarization data - interstellar magnetic field: } \\
\text { Galactic }\end{array}$} & $38^{\circ}$ \\
Ecliptic & $263^{\circ}$ & $23^{\circ}$ \\
Center of Ribbon arc: & & $37^{\circ}$ \\
Galactic & $33^{\circ}$ & \\
Ecliptic $^{3}$ & $221^{\circ}$ & $55^{\circ}$ \\
\hline
\end{tabular}

${ }^{1}$ Galactic coordinates are denoted by $\ell, b$, and ecliptic coordinates by $\lambda, \beta$. The estimated uncertainties on the best fit direction are $\pm 35^{\circ}$, based on the broad minimum for the best-fit function, $F_{\mathrm{i}}$.

${ }^{2}$ This direction makes an angle of $\sim 71^{\circ}$ with respect to the vector motion of the flow of ambient local ISM past the Sun, in the LSR, which is from $\ell, b=331^{\circ},-5^{\circ}$ with velocity of $-19.4 \mathrm{~km} \mathrm{~s}^{-1}$ (Frisch \& Slavin 2006).

${ }^{3}$ This direction makes an angle of $\sim 46^{\circ}$ with respect to the heliocentric vector motion of the flow of interstellar $\mathrm{He}^{\circ}$ into the heliosphere, which is from $\lambda, \beta \sim 255^{\circ}, 5^{\circ}$ with velocity of $-26.3 \mathrm{~km} \mathrm{~s}^{-1}$ (Witte 2004). 
stars with ecliptic latitudes $\beta>+10^{\circ}$ consistently show much smaller polarizations than stars with ecliptic latitudes below $+10^{\circ}$. This statement is also nearly true when galactic latitudes are used instead. All stars with polarizations less than $0.01 \%$ have ecliptic latitudes greater than $\beta=10^{\circ}$. All stars with polarizations larger than $0.01 \%$, except for HD 150997, are located at more negative latitudes, $\beta<10^{\circ}$. This effect follows from the distribution of ISM very close to the Sun, within $15 \mathrm{pc}$, which has higher column densities towards negative galactic latitudes than towards positive galactic latitudes in the galactic center hemisphere (e.g. Frisch et al. 2009). The ecliptic latitude of HD 150997 is $+60^{\circ}$, and it is $26^{\circ}$ from the ISMF pole at $\ell=38^{\circ}, b=23^{\circ}$. A single isolated clump of dust towards HD 150997 is possible, or the polarization may be intrinsic to the stellar system. In Fig. 5 the stars used in the fit are color-coded according to the data source. Other significant data points, with polarizations larger than the $3 \sigma$ data uncertainties but not used, in the fit are plotted as open squares.

Based on the above discussions we estimate uncertainties of $\pm 35^{\circ}$ on the best-fit ISMF direction of $\lambda, \beta \sim 263^{\circ}, 37^{\circ}$ in ecliptic coordinates, or $\ell, b \sim 38^{\circ}, 23^{\circ}$ in galactic coordinates. This direction is $33^{\circ}$ from the ISMF direction at the heliosphere determined from the arc of the IBEX Ribbon.

\section{Discussion}

The ISMF direction of $\ell, b \sim 38^{\circ}, 23^{\circ}$, found from local polarization data, is directed towards the tangential region of Loop I at the North Polar Spur (NPS), that rises $50^{\circ}$ vertically from the galactic plane near $\ell \sim 30^{\circ}-40^{\circ}$. The NPS is a region of radio intense synchrotron emission, that Wolleben (2007) attributes to the collision of the S1 and S2 subshells of Loop I. Positive Faraday rotation measures for distant pulsars and extragalactic sources towards the NPS indicate an ISMF that is directed towards the Sun (Taylor et al. 2009). However at the southern galactic latitudes for the same longitudes, where Wolleben's model suggests the S1 subshell has expanded to the solar location, Faraday rotation measures are negative as is consistent with an ISMF direction pointing away from the Sun and towards the azimuthal field direction of $\ell \sim 83^{\circ}$. Salvati (2010) analyzed Faraday rotation measure and dispersion data towards four pulsars, 150-300 pc away in the low density third galactic quadrant, and found an ISMF directed towards $\ell, b=5^{\circ}, 42^{\circ}$, with strength $3.3 \mu \mathrm{G}$, and with polarity directed into the northern galactic hemisphere. These data give the ISMF in the downwind direction, and it is within $33^{\circ}$ of the best-fitting polarization ISMF direction and within $22^{\circ}$ of the ISMF direction indicated by the center of the Ribbon arc. The good agreement between these three independent methods of obtaining the ISMF direction suggests the three kinds of measurements are tracing the same ISMF, and that it is relatively smooth.

The formation mechanism for the Ribbon must be understood in order to relate the magnetic 
field direction derived from the Ribbon configuration, with the magnetic field derived from polarization data. The $26.3 \mathrm{~km} \mathrm{~s}^{-1}$ relative motion between the heliosphere and circumheliosphere ISM displaces the ISMF so that it drapes over the heliosphere, and the geometry of the outer heliosphere depends on the angle between the ISMF and interstellar flow vectors (e.g. Ratkiewicz et al. 2000; Pogorelov et al. 2009; Opher et al. 2009; Izmodenov 2009). The center of the Ribbon arc defines a magnetic field direction that makes an angle of $\sim 46^{\circ}$ with the gas flow vector (Table 1). Heerikhuisen et al. (2010) reproduce the Ribbon figuration using three-dimensional MHD models of the heliosphere plasma, coupled to interstellar neutrals described by a kinetic distribution, and ions with a Lorentzian distribution. In this model, the ENAs originate upstream of the heliopause in the region where the ISMF angle varies with the distance beyond the heliopause. The model reproduces the location of the IBEX Ribbon quite well for the ISMF direction of $\lambda, \beta=224^{\circ}, 41^{\circ}$, which is close to the center of the Ribbon arc. The details of the Heerikhuisen et al. model are not yet substantiated, because the pitch-angle distribution of the underlying pickup ion ring-beam may scatter over timescales shorter than the charge-exchange lifetime (McComas et al. 2009a; Florinski et al. 2010). These same MHD models also show that the Ribbon moves towards the equator of the distant ISMF as the ENA origin pushes further upstream of the heliopause. Several alternative scenarios have been discussed for the Ribbon formation, including an origin in the inner heliosheath (McComas et al. 2009a; Schwadron et al. 2009; McComas et al. 2010). Comparisons between magnetic field directions derived from the Ribbon arc and polarization data will yield information on large-scale magnetic turbulence in the solar vicinity, once the formation of the Ribbon is fully understood.

The ISM towards the star $36 \mathrm{Oph}\left(5 \mathrm{pc}\right.$ away and $10^{\circ}$ from the heliosphere nose) provides insights into the ISMF and gas forming the polarizations observed in the upwind direction. Tinbergen (1982) observed a polarization of $\sim 0.02 \%$ towards $36 \mathrm{Oph}$, a strength that is unusually high compared to the mean polarizations found over long sightlines. A single interstellar cloud, the ' $G$ ' cloud, is present in front of both $36 \mathrm{Oph}(5 \mathrm{pc})$ and the nearest star $\alpha$ Cen $(1.3 \mathrm{pc})$, with a velocity that differs by $2 \mathrm{~km} \mathrm{~s}^{-1}$ from the ISM velocity inside of the heliosphere (e.g. Frisch et al. 2009, and references therein). This suggests that there is a single polarization screen within $1.3 \mathrm{pc}$ of the Sun in the upwind direction, and allows the possibility that the ISMF direction derived from the polarization data is sampling a different magnetic field than the Ribbon arc. Heliosphere models predict that the configuration of the IBEX Ribbon is sensitive to $20^{\circ}$ variations in the direction of the ISMF, so that a solar transition between the two field directions should be readily apparent in the configuration of the IBEX ribbon (Frisch et al. 2010). The Ribbon models are not yet proven, and the uncertainties on the ISMF direction obtained from the polarization data are large. Nevertheless, it appears that the different ISMF directions obtained from the center of the Ribbon arc and starlight polarizations can provide information on the true large scale variations in the local ISMF, and ultimately on small-scale magnetic turbulence. 
The local ISMF direction derived from polarization data can be used to test the possibility that the ISMF is perpendicular to the flow of ISM past the Sun. The interstellar cloud surrounding the heliosphere is part of a cluster of local interstellar cloudlets (CLIC) that has a mean flow velocity directed away from the center of the Loop I magnetic superbubble (e.g. Frisch et al. 2009). For comparing the kinematical CLIC with the geometrically defined configuration of Loop I, the interstellar velocities are first converted into the local standard of rest (LSR). The standard LSR conversion generally assumed for converting radio velocities to the LSR is used here, e.g. a solar apex velocity of $19.5 \mathrm{~km} \mathrm{~s}^{-1}, 56^{\circ}, 23^{\circ}$. The result is an LSR upwind direction for the CLIC that is towards $\ell, b=331^{\circ},-5^{\circ}$, and an LSR upwind direction for the circumheliospheric cloud that is directed towards $\ell, b=318^{\circ}, 0^{\circ}$. Both of these directions are toward the central regions of Loop I. A value of $\sim 71^{\circ}$ is found for the angle between the mean LSR flow velocity vector of the CLIC and the best-fitting ISMF direction from polarization data, $\ell, b \sim 38^{\circ}, 23^{\circ}$. The ISMF direction is therefore nearly perpendicular to the cloud motions, similar to what might be expected for the expansion of an evolved superbubble shell in pressure equilibrium with ambient ISM. For the ISM around the heliosphere, the angle between the ISMF direction from the Ribbon arc and the heliocentric $\mathrm{He}^{\circ}$ flow vector is $\sim 46^{\circ}$. The spatial regions sampled by starlight polarization and CLIC interstellar absorption features are similar, since stars within 30-40 pc of the Sun are utilized in both comparisons. Additional information on magnetic turbulence in the flow of ISM past the Sun might be obtained from the Chandrasekhar-Fermi method, by comparing the velocity dispersion of clouds with the dispersion of polarization position angles (e.g. Andersson \& Potter 2006).

There may be some contribution to the polarizations from interstellar grains in the outer heliosheath, which will tightly follow the ISMF that interacts with and is deflected around the heliosphere. The magnetic field upstream of the heliopause filters out grains with large charge-to-mass ratios, $\mathrm{Q} / \mathrm{M}$, and gyroradii that are smaller than the characteristic lengths between the heliosphere bow shock and heliopause (e.g., Slavin et al. 2009). The radii of the magnetically excluded grains are $<0.01-0.1 \mu \mathrm{m}$, and are comparable to the sizes of interstellar polarizing grains (e.g. Mathis 1986). The strongest polarizations occur for stars located on the northern edge of the IBEX Ribbon, which is $15^{\circ}-30^{\circ}$ above the equator of the ISMF direction towards the arc center, corresponding to the blue points in Fig. 5. For truly interstellar polarization and a uniform dust distribution, the strongest polarizations are expected at the equator of the ISMF, so perhaps additional contributions to starlight polarizations from nano-sized grains in the heliosheath regions are possible.

The local ISMF direction found here has a curious coincidence with the CMB dipole (as found previously, Frisch 2007). The great circle midway between hot and cold poles of the cosmic microwave background dipole moment bifurcates the heliosphere nose region and is aligned with the direction of the local ISMF direction found here, to within the uncertainties. The ISMF direction of $\ell, b \sim 38^{\circ}, 23^{\circ}$ is at an angle of $90^{\circ} \pm 8^{\circ}$ from the dipoles at $\ell, b=264^{\circ},+48^{\circ}$ and $83^{\circ},-48^{\circ}$. 


\section{Conclusions}

In this exploratory study we find the best-fit to the polarization position angles towards $\sim 30$ stars within $40 \mathrm{pc}$ of the Sun and $90^{\circ}$ of the heliosphere nose $\left(16^{\circ}\right.$ above the galactic center), using mainly data from the literature, is $\ell, b \sim 38^{\circ}, 23^{\circ}$ (or $\lambda, \beta \sim 263^{\circ}, 37^{\circ}$, Table 1 ), with uncertainties of $\pm 35^{\circ}$ based on the flat minimum of the best fit direction. The ISMF direction indicated by the center of the IBEX Ribbon arc is $\ell, b \sim 33^{\circ}, 55^{\circ}$. The difference between the two, $\sim 33^{\circ}$, is marginally significant given the uncertainties. The sensitivity of the IBEX Ribbon to variations of $\leq 20^{\circ}$ in the ISMF direction, and the fact that the Sun is in a cloud that is different from the nearest upwind ISM, support the possibility that the different ISMF directions obtained from the Ribbon and polarization data are tracking either large-scale distortion of the magnetic field direction, or possibly small-scale magnetic turbulence. This comparison is possible because the Ribbon is observed where the ISMF draping over the heliosphere is perpendicular to the sightline. Better constraints on the distortion of the ISMF in the solar vicinity will be possible once the formation of the ENA Ribbon is understood, and more high sensitivity polarization data towards the Ribbon are available.

There are several implications of the ISMF direction found here. The ISMF vector direction is perpendicular to the bulk LSR velocity of the cluster of local interstellar clouds flowing past the Sun, which is consistent with an origin in an evolved expanding magnetized superbubble shell. The similarity of the ISMF directions found from the ENA Ribbon and optical polarization data suggests that the nearby ISMF is coherent over scale sizes of decades in parsecs, and that variations in the ISMF direction due to large scale distortion or magnetic turbulence are on the order of $35^{\circ}$ or smaller. A curious coincidence is that the direction of the local ISMF is within $8^{\circ}$ of the great circle that divides the hot and cold poles of the cosmic microwave background dipole moment, which also passes through the heliosphere nose.

Acknowledgements: This research has been supported by NASA grants NNX09AH50G and NNX08AJ33G to the University of Chicago, and by the IBEX mission as a part of NASA's Explorer Program. We would like to thank Philip Lucas for sharing PlanetPol data prior to publication. 


\section{REFERENCES}

Andersson, B.-G. \& Potter, S. B. 2006, ApJ, 640, L51

Appenzeller, I. 1968, ApJ, 151, 907

Bailey, J., Lucas, P. W., \& Hough, J. H. 2010, MNRAS, 586

Barstow, M. A., Dobbie, P. D., Holberg, J. B., Hubeny, I., \& Lanz, T. 1997, MNRAS, 286, 58

Davis, L. 1955, Physical Review, 100, 1440

Davis, Jr., L. \& Berge, G. L. 1968, in Nebulae and Interstellar Matter (the University of Chicago Press), 755-+

Florinski, V., Zank, G., Hu, Q., Heerikhuisen, J., Hu, Q., \& Khazanov, I. 2010, ApJ, 718, 1097

Frisch, P. C. 2007, ArXiv e-prints:arXiv:0707.2970v2

-. 2010, ApJ, 714, 1679

Frisch, P. C., Bzowski, M., Grün, E., Izmodenov, V., Krüger, H., Linsky, J. L., McComas, D. J., Möbius, E., Redfield, S., Schwadron, N., Shelton, R. R., Slavin, J. D., \& Wood, B. E. 2009, Space Sci. Rev., 28

Frisch, P. C., Heerikhuisen, J., Pogorelov, N. V., DeMajistre, B., Crew, G. B., Funsten, H. O., Janzen, P. H., McComas, D. J., Moebius, E., Mueller, H.-R., Reisenfeld, D. B., Schwadron, N. A. Slavin, J. D., \& Zank, G. P. 2010, ApJ, 719, 1984

Frisch, P. C. \& Slavin, J. D. 2006, Short Term Variations in the Galactic Environment of the Sun, in Solar Journey: The Significance of Our Galactic Environment for the Heliosphere and Earth, Ed. P. C. Frisch (Springer), 133-193

Funsten, H. O., Allegrini, F., Crew, G. B., DeMajistre, R., Frisch, P. C., Fuselier, S. A., Gruntman, M., Janzen, P., McComas, D. J., Möbius, E., Randol, B., Reisenfeld, D. B., Roelof, E. C., \& Schwadron, N. A. 2009, Science, 326, 964

Fuselier, S. A., Allegrini, F., Funsten, H. O., Ghielmetti, A. G., Heirtzler, D., Kucharek, H., Lennartsson, O. W., McComas, D. J., Möbius, E., Moore, T. E., Petrinec, S. M., Saul, L. A., Scheer, J. A., Schwadron, N., \& Wurz, P. 2009, Science, 326, 962

Heerikhuisen, J., Pogorelov, N. V., Florinski, V., Zank, G. P., \& le Roux, J. A. 2008, ApJ, 682, 679

Heerikhuisen, J., Pogorelov, N. V., Zank, G. P., Crew, G. B., Frisch, P. C., Funsten, H. O., Janzen, P. H., McComas, D. J., Reisenfeld, D. B., \& Schwadron, N. A. 2010, ApJ, 708, L126 
Heiles, C. 1976, ARA\&A, 14, 1

—. 1989, ApJ, 336, 808

-. 1996, ApJ, 462, 316

-. 1998, ApJ, 498, 689

Heiles, C. 2009, in ArXiv Astrophysics e-prints, Vol. 2010, astro2010: The Astronomy and Astrophysics Decadal Survey, 121-+

Holzer, T. E. 1989, ARA\&A, 27, 199

Izmodenov, V. V. 2009, Space Science Reviews, 143, 139

Izmodenov, V. V., Malama, Y. G., Ruderman, M. S., Chalov, S. V., Alexashov, D. B., Katushkina, O. A., \& Provornikova, E. A. 2009, Space Science Reviews, 146, 329

Lallement, R., Quémerais, E., Bertaux, J. L., Ferron, S., Koutroumpa, D., \& Pellinen, R. 2005, Science, 307,1447

Lazarian, A. 2003, Journal of Quantitative Spectroscopy and Radiative Transfer, 79, 881

Leroy, J. L. 1993, A\&AS, 101, 551

Mathis, J. S. 1986, ApJ, 308, 281

McComas, D. J., Allegrini, F., Bochsler, P., Bzowski, M., Christian, E. R., Crew, G. B., DeMajistre, R., Fahr, H., Fichtner, H., Frisch, P. C., Funsten, H. O., Fuselier, S. A., Gloeckler, G., Gruntman, M., Heerikhuisen, J., Izmodenov, V., Janzen, P., Knappenberger, P., Krimigis, S., Kucharek, H., Lee, M., Livadiotis, G., Livi, S., MacDowall, R. J., Mitchell, D., Möbius, E., Moore, T., Pogorelov, N. V., Reisenfeld, D., Roelof, E., Saul, L., Schwadron, N. A., Valek, P. W., Vanderspek, R., Wurz, P., \& Zank, G. P. 2009a, Science, 326, 959

McComas, D. J., Allegrini, F., Bochsler, P., Bzowski, M., Collier, M., Fahr, H., Fichtner, H., Frisch, P., Funsten, H. O., Fuselier, S. A., Gloeckler, G., Gruntman, M., Izmodenov, V., Knappenberger, P., Lee, M., Livi, S., Mitchell, D., Möbius, E., Moore, T., Pope, S., Reisenfeld, D., Roelof, E., Scherrer, J., Schwadron, N., Tyler, R., Wieser, M., Witte, M., Wurz, P., \& Zank, G. 2009b, Space Science Reviews, 146, 11

McComas, D. J., Bzowski, M., Frisch, P. C., Crew, G. B., Dayeh, M. A., DeMajistre, R., Funsten, H. O., Fuselier, S. A., Gruntman, M., Janzen, P., Kubiac, M. A., Livadiotis, G., Möbius, E., Reisenfeld, D., \& Schwadron, N. A. 2010, J. Geophys. Res., "in press", 00, 00 
Möbius, E., Bochsler, P., Bzowski, M., Crew, G. B., Funsten, H. O., Fuselier, S. A., Ghielmetti, A., Heirtzler, D., Izmodenov, V. V., Kubiak, M., Kucharek, H., Lee, M. A., Leonard, T., McComas, D. J., Petersen, L., Saul, L., Scheer, J. A., Schwadron, N., Witte, M., \& Wurz, P. 2009, Science, 326, 969

Opher, M., Richardson, J. D., Toth, G., \& Gombosi, T. I. 2009, Space Science Reviews, 143, 43

Parker, E. N. 1961, ApJ, 134, 20

Piirola, V. 1977, A\&AS, 30, 213

Piirola, V., Berdyugin, A., Mikkola, S., \& Coyne, G. V. 2005, ApJ, 632, 576

Pogorelov, N. V., Heerikhuisen, J., Zank, G. P., \& Borovikov, S. N. 2009, Space Science Reviews, 143,31

Pogorelov, N. V., Zank, G. P., \& Ogino, T. 2004, ApJ, 614, 1007

Ratkiewicz, R., Barnes, A., \& Spreiter, J. R. 2000, J. Geophys. Res., 105, 25021

Ratkiewicz, R. \& Grygorczuk, J. 2008, Geophys. Res. Lett., 35, 23105

Salvati, M. 2010, A\&A, 513, A28+

Schwadron, N. A., Bzowski, M., Crew, G. B., Gruntman, M., Fahr, H., Fichtner, H., Frisch, P. C., Funsten, H. O., Fuselier, S., Heerikhuisen, J., Izmodenov, V., Kucharek, H., Lee, M., Livadiotis, G., McComas, D. J., Moebius, E., Moore, T., Mukherjee, J., Pogorelov, N. V., Prested, C., Reisenfeld, D., Roelof, E., \& Zank, G. P. 2009, Science, 326, 966

Slavin, J. D. \& Frisch, P. C. 2008, A\&A, 491, 53

Slavin, J. D., Frisch, P. C., Heerikhuisen, J., Pogorelov, N. V., Mueller, H., Reach, W. T., Zank, G. P., Dasgupta, B., \& Avinash, K. 2009, Space Science Reviews, (Proceedings of Solar Wind 12). in press (http://adsabs.harvard.edu/abs/2009arXiv0911.1492S)

Stone, E. C., Cummings, A. C., McDonald, F. B., Heikkila, B. C., Lal, N., \& Webber, W. R. 2008, Nature, 454, 71

Swisdak, M., Opher, M., Drake, J. F., \& Alouani Bibi, F. 2010, ApJ, 710, 1769

Taylor, A. R., Stil, J. M., \& Sunstrum, C. 2009, ApJ, 702, 1230

Tinbergen, J. 1982, A\&A, 105, 53

Wiktorowicz, S. J. \& Matthews, K. 2008, PASP, 120, 1282 
Witte, M. 2004, A\&A, 426, 835

Wolleben, M. 2007, ApJ, 664, 349

This preprint was prepared with the AAS LATEX macros v5.2. 


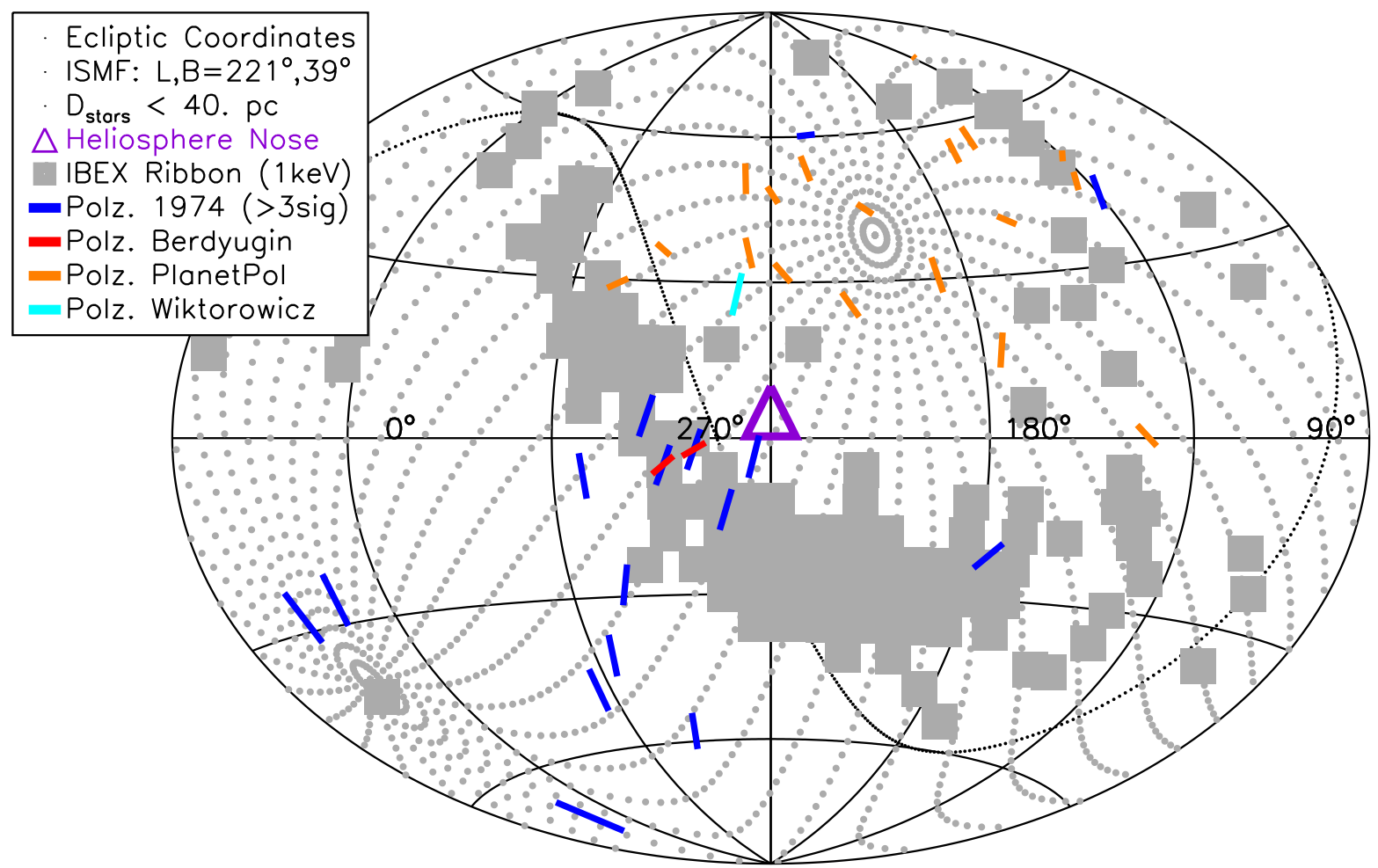

Fig. 1.- The polarization vectors of stars within $\sim 40 \mathrm{pc}$ are shown in the ecliptic coordinate system, and color-coded for the data source. The '1974' data are from Tinbergen (1982, collected in 1974) and Piirola (1977). The plot is centered on the heliosphere nose located at ecliptic coordinates (purple triangle) of $\lambda=255.4^{\circ}, \beta=5.1^{\circ}$, and longitude increases towards the left in each figure. Symbol sizes do not indicate the strength of the polarization. The Compton-Getting corrected ENA fluxes at $1 \mathrm{keV}$ are plotted for directions where the ENA count rates are larger than 113 counts $\mathrm{cm}^{-2} \mathrm{~s}^{-1} \mathrm{sr}^{-1} \mathrm{keV}^{-1}$, which is 1.5 times the mean ENA flux at $1 \mathrm{keV}$ as measured by the IBEX-HI instrument (McComas et al. 2009a). The dotted lines show the ISMF determined by the center of the Ribbon arc (Funsten et al. 2009). 


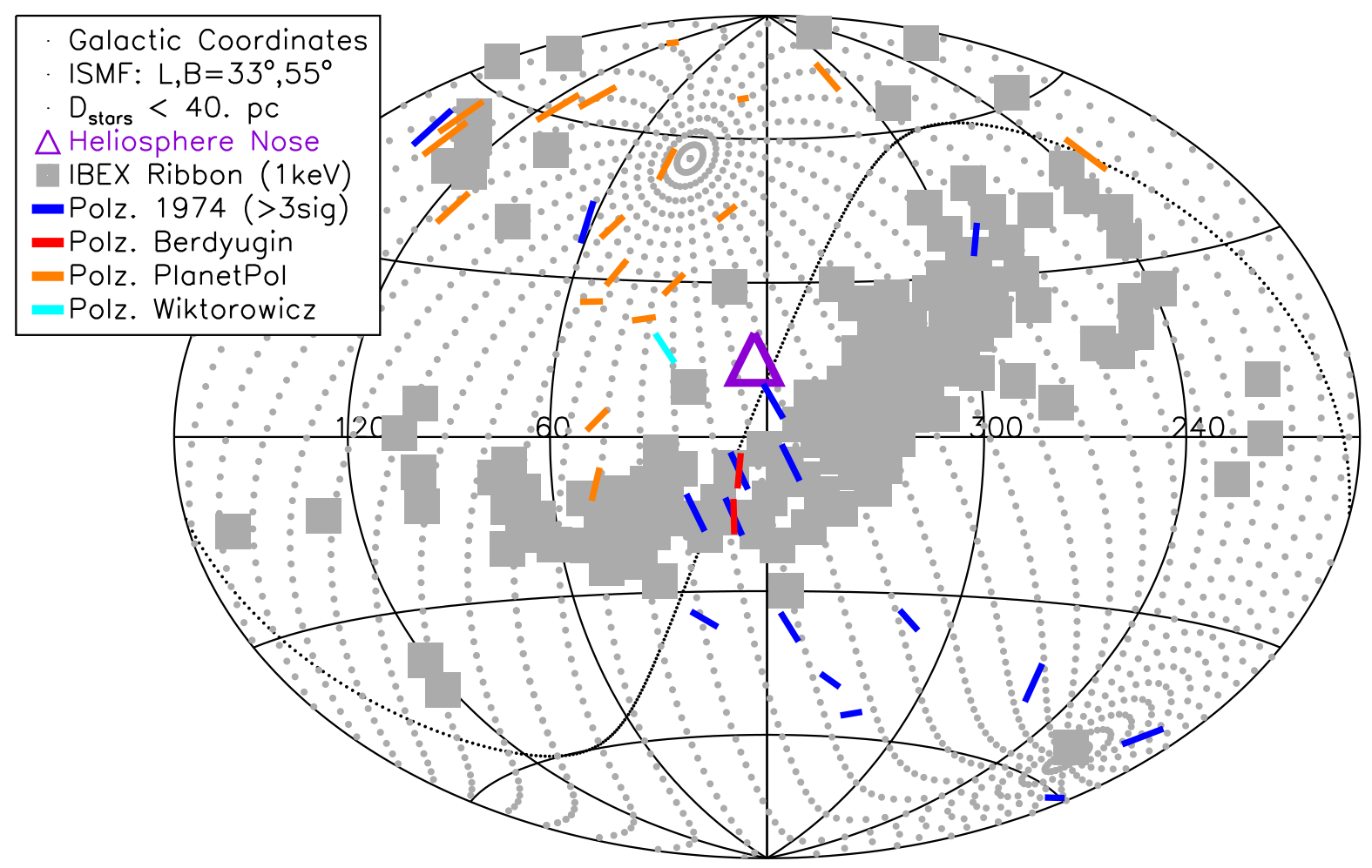

Fig. 2.- Same as Fig. 1, except the figure is in galactic coordinates, and centered on the galactic center. 


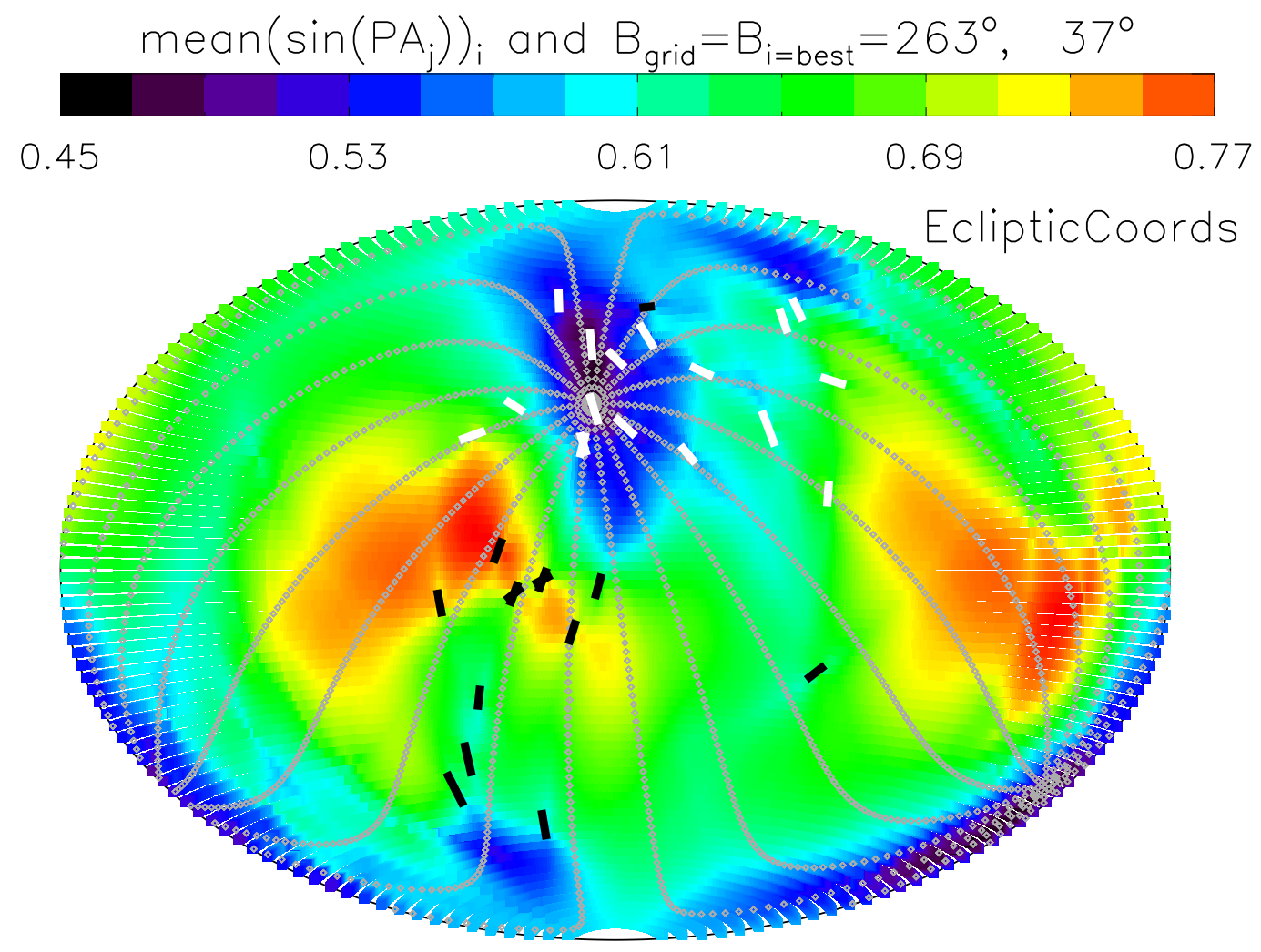

Fig. 3.- The value of the function $F_{\mathrm{i}}=\overline{\sin \left(\theta_{\mathrm{j}, \mathrm{i}}\right)}$ evaluated over a regular grid of $i$ possible interstellar field directions (see $\$ 2$ ). The function is color-coded and plotted in the ecliptic coordinate system, centered on the ecliptic nose, at $\lambda \sim 255^{\circ}$. The gray dotted grid shows the best-fit in the ecliptic coordinate system to the ISMF, $B_{\mathrm{i}=\text { best }}$, which is directed towards $\lambda, \beta=263^{\circ}, 37^{\circ}$. Polarization vectors for the stars used in the fit are shown as either black or white bars, for visual clarity. 


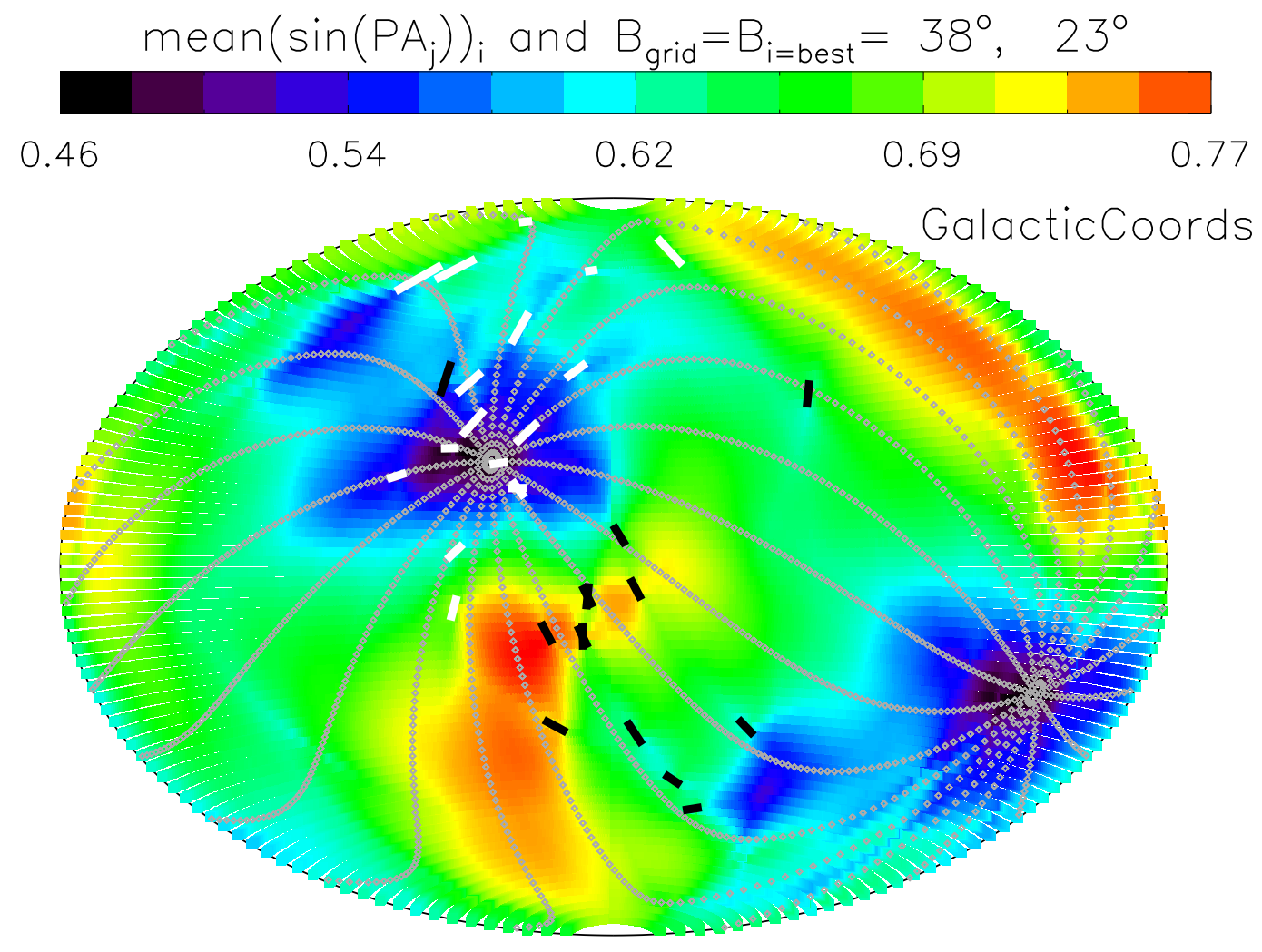

Fig. 4.- Same as Fig. 3, except quantities are plotted in galactic coordinates and centered on the galactic center. The best fit to the ISMF in the galactic coordinate system, $B_{\mathrm{i}=\text { best }}$, is directed towards $\ell, b=38^{\circ}, 23^{\circ}$. 


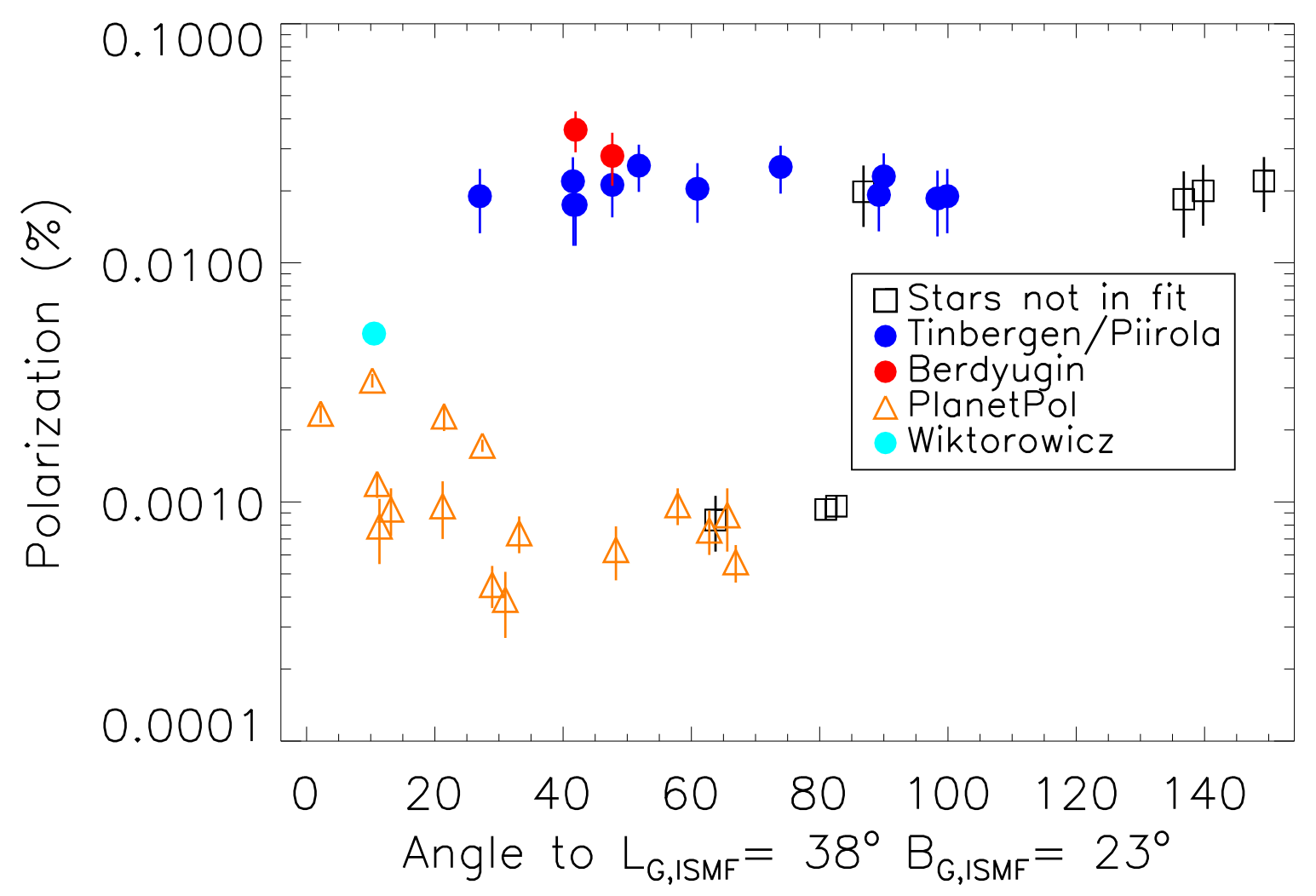

Fig. 5.- The polarizations of stars in the combined sample (in units of percentage of degree of polarization, ordinate) plotted against the angular distance between the star and the north pole of the best-fitting interstellar magnetic field, which is $\ell \sim 38^{\circ}, b \sim 23^{\circ}$. The stars whose position angles are used to calculate the magnetic field direction are plotted as dots or triangles, and color coded to indicate the origin of the data. The stars in the full sample that have polarizations larger than the $3 \sigma$ uncertainties, but that were not used in the position angle fit (see text), are plotted as open squares. All stars with polarizations less than $0.01 \%$ have ecliptic latitudes greater than $\beta=10^{\circ}$, while all stars with polarizations larger than $0.01 \%$, except for HD 150997, are located at more negative latitudes, $\beta<10^{\circ}$. The ecliptic latitude of HD 150997 is $+60^{\circ}$, and it is $26^{\circ}$ from the ISMF pole at $\ell=38^{\circ}, b=23^{\circ}$. 